\title{
CARACTERIZAÇÃO E CLASSIFICAÇÃO DE SOLOS DESENVOLVIDOS DE ARENITOS DA FORMAÇÃO AQUIDAUANA-MS ${ }^{(1)}$
}

\author{
Jolimar Antonio Schiavo ${ }^{(2)}$, Marcos Gervásio Pereira ${ }^{(3)}$, Luiz Paulo \\ Montenegro de Miranda ${ }^{(4)}$, Antonino Hypólito Dias Neto ${ }^{(5)} \&$ \\ Ademir Fontana ${ }^{(6)}$
}

\begin{abstract}
RESUMO
A formação Aquidauana é constituída por um conjunto de sedimentos com até $500 \mathrm{~m}$ de espessura, predominando arenito de granulometria fina a média, intercalado com conglomerado arenoso. Nesse ambiente, o relevo é um dos principais fatores condicionantes na formação de solos. Objetivou-se, neste trabalho, caracterizar e classificar os solos desenvolvidos desses arenitos. Para isso, foram estudados quatro perfis ao longo de uma topossequência, de uma pendente representativa das colinas suaves onduladas verificadas na área de estudo. Os perfis localizavam-se no terço superior (P1), terço médio (P2), terço inferior (P3) e sopé de encosta (P4). Eles foram morfologicamente descritos, e os horizontes, caracterizados quanto às propriedades físicas e químicas. Os solos estudados apresentaram predomínio da fração areia $\left(>680 \mathrm{~g} \mathrm{~kg}^{-1}\right)$, com textura variando de franco-arenosa ( $(\mathrm{P} 4)$ a franco-argiloarenosa. Os valores de $\mathrm{pH}$ em água variaram de 4,2 a 6,5 . Os valores de capacidade de troca catiônica variaram de $1,6 \mathrm{cmol}_{\mathrm{c}} \mathrm{kg}^{-1}$ no P4 a 10,3 $\mathrm{cmol}_{\mathrm{c}} \mathrm{kg}^{-1}$ no P2, com predomínio dos íons $\mathrm{H}^{+}$no P1 e P4 e Ca ${ }^{2+}$ no P2 e P3. Os horizontes subsuperficiais do P1 e P4 são distróficos, enquanto em P2 e P3 verificou-se elevada saturação por bases, evidenciando caráter eutrófico. À exceção do P2, os demais apresentaram argila de baixa atividade. Em todos os perfis verificaram-se atributos morfológicos, físicos, químicos e mineralógicos condicionados pelo material de origem e relevo, demonstrando a influência desses fatores na pedogênese.
\end{abstract}

Termos de indexação: SiBCS, taxonomia de solos, Bioma Cerrado Pantanal.

\footnotetext{
(1) Recebido para publicação em julho de 2009 e aprovado em março de 2010.

(2) Professor Associado IV da Universidade Estadual de Mato Grosso do Sul - UEMS. Rodovia Aquidauana, km 12, CEP 79200000 Aquidauana (MS). E-mail: schiavo@uems.br

(3) Professor Associado II do Departamento de Solos da Universidade Federal Rural do Rio de Janeiro, BR 465, km 7, CEP 23890000 Seropédica (RJ). Bolsista do CNPq e FAPERJ. E-mail: gervasio@ufrrj.br

(4) Mestrando do Programa de Pós-Graduação em Agronomia, Universidade Estadual Paulista "Julio de Mesquita Filho". Av. Brasil 56, Centro, CEP 15385-000 Campus de Ilha Solteira (SP). E-mail: luizpauloagro@yahoo.com.br

(5) Mestrando do Programa de Pós-Graduação em Agronomia da UEMS. E-mail: tonybioagro@hotmail.com

${ }^{(6)}$ Doutor em Agronomia - Ciência do Solo, Engenheiro-Agrônomo do INCRA. Av. Senador Robert Kennedy, São Torquato, CEP 29114-300 Vila Velha, (ES). E-mail: ademir.fontana@gmail.com
} 


\title{
SUMMARY: CHARACTERIZATION AND CLASSIFICATION OF SOILS DEVELOPED FROM SANDSTONE OF THE AQUIDAUANA FORMATION
}

\begin{abstract}
The Aquidauana geological formation consists of a set of up to $500 \mathrm{~m}$ thick sediments predominantly composed of sandstone with fine to medium grain size, interspersed with sandy conglomerates. In this environment, the topography is one of the main conditioning factors of the formation of different soil types in the landscape. The objective of this study was to characterize and classify soils developed from these sandstones. Four soils profiles along a representative toposequence of gentle slopes were sampled and studied. The profiles were located on the shoulder (P1), backslope (P2), footslope (P3) and toeslope (P4). The soil profiles were morphologically described and physical and chemical properties of their horizons evaluated. The sand fraction was predominant in all profiles $\left(>680 \mathrm{~g} \mathrm{~kg}^{-1}\right)$, with textures ranging from sandy loam (P4) to clay sandy loam. The water $p H$ values ranged from 4.2 to 6.5. The cation exchange capacity values ranged from $1.6 \mathrm{cmol}_{c} \mathrm{~kg}^{-1}$ (in P4) to $10.3 \mathrm{cmol}_{c} \mathrm{~kg}^{-1}$ (in P2), with a predominance of hydrogen ions in $P 1$ and $P 4$, and calcium in profile P2 and P3. The $P 1$ and $P 4$ pedons were dystrophic, whereas in $P 2$ and $P 3$, a high base saturation status evidenced an eutrophic character. Except in P2, the profiles presented low activity clays. In all soil profiles the morphological, physical, chemical and mineralogical properties were conditioned by the parent material and relief, demonstrating the influence of these factors on the pedogenesis.
\end{abstract}

Index terms: SiBCS, soil taxonomy, Cerrado Pantanal Biome.

\section{INTRODUÇÃO}

A formação Aquidauana, datada do período Carbonífero Superior, localizada em Mato Grosso do Sul compreende arenitos com granulometria variável de fina a grosseira, com ampla gama de cores, desde avermelhadas, cinza arroxeadas até esbranquiçadas. A formação está assentada sobre rochas PréCambrianas representadas pelo Grupo Cuiabá e sequências rudimentares Paleozoicas dos Grupos Paraná (Formação Furnas e formação Aquidauana), compostas por arenitos porosos e friáveis (RADAMBRASIL, 1982).

Essa formação geológica insere-se na transição entre os platôs resquiciais do Planalto Central e a Planície do Pantanal, sendo a maior parte de seus solos formada por arenitos e quartzitos. O relevo escarpado circunscreve platôs com níveis acentuados de elevação posicionados a leste-noroeste do município de Aquidauana. Descrita como a porção superior do relevo denominado Grupo Arenito Aquidauana, é semelhante à formação Botucatu, porém originária de ambiente flúvio-lacustre e apresentando aproximadamente $500 \mathrm{~m}$ de espessura.

A melhor representação dos níveis clásticos dessa formação está na posição setentrional devido ao intemperismo mais acentuado, sendo verificados afloramentos de arenitos mais preservados (RADAMBRASIL, 1982). Nos arenitos da formação Aquidauana são comuns feições ferruginosas, cuja distribuição e formas de ocorrência são decorrentes dos fatores e processos que atuaram durante a evolução das paisagens atuais. No entanto, estudos da evolução e caracterização de solos em função do relevo neste ambiente de formação são bastante escassos.

Informações a respeito da distribuição de solos na região da interface do bioma Cerrado Pantanal, baseadas principalmente em levantamentos de solos generalizados, dada a carência de trabalhos detalhados ou semidetalhados na região, permitem concluir que os solos possuem grande heterogeneidade. Apesar de a maior parte dos solos dessa região ser descrita como profundos, com baixo conteúdo de nutrientes e ausência de minerais primários facilmente intemperizáveis e localizados em áreas planas a suave onduladas, ocorrem também solos rasos, principalmente Cambissolos e Neossolos Litólicos, situados em áreas acidentadas, que podem ser tanto álicos como por vezes eutróficos (Motta et al., 2002). Nas áreas mais acidentadas também podem ser encontrados solos eutróficos, como Argissolos e Nitossolos, geralmente associados a uma maior influência de um material de origem mais básico (Motta et al., 2002).

Nesse ambiente, onde predominam as formas escarpadas, a participação do relevo na evolução dos solos tem importância destacada, visto que ele influencia a quantidade de água que pode infiltrar, acelerando as reações químicas do intemperismo, ou escorrer por superfície, promovendo o transporte de sólidos ou de materiais em solução, com efeitos que se traduzem na formação de diferentes solos, nas diversas 
posições das topossequências (Curi \& Franzmeier, 1984; Motta et al., 2002). Associada à dinâmica da água na modelagem da paisagem, a erosão geológica dessas áreas provocada pelo recuo das encostas também contribui para a evolução dos solos.

Em estudo de topossequências de Latossolos originados de rochas basálticas no Paraná, Ghidin et al. (2006) observaram que a posição dos perfis na paisagem foi determinante na definição da mineralogia da fração argila. Para os perfis em estudo, foi verificado o predomínio de gibbsita e hematita, indicando que as condições topográficas foram favoráveis para que as reações de hidrólise ocorressem de maneira mais intensa, contribuindo para o desenvolvimento da mineralogia oxídica. Ainda, esses autores verificaram que no ponto mais baixo da paisagem, devido à drenagem limitada, há maior presença de óxidos de Fe de baixa cristalinidade. Especificamente, em estudo de caracterização e gênese de perfis com horizontes plínticos desenvolvidos de arenito Bauru, Coelho \& Vidal Torrado (2003) verificaram maior concentração de $\mathrm{Fe}$ total nas petroplintitas, sendo compostos essencialmente de óxidos e oxi-hidróxidos de $\mathrm{Fe}$ cristalinos, ao passo que nas plintitas observaram-se maiores teores dos compostos de Fe de baixa cristalinidade.

Este trabalho teve como objetivo avaliar a influência do fator relevo nas características morfológicas, físicas e químicas de perfis de solos ao longo de uma topossequência do Arenito da formação Aquidauana (MS).

\section{MATERIAL E MÉTODOS}

A área de estudo localiza-se no município de Aquidauana, Mato Grosso do Sul, entre as coordenadas $20^{\circ} 27^{\prime} 20^{\prime \prime}$ S, $55^{\circ} 40^{\prime} 17 "$ W e altitude de aproximadamente $168 \mathrm{~m}$. Segundo Köppen, o clima pertence ao tipo Aw (tropical úmido), com precipitação pluvial média anual de $1.200 \mathrm{~mm}$ e temperaturas máximas e mínimas de 33 e $19^{\circ} \mathrm{C}$, respectivamente. $\mathrm{O}$ material de origem é proveniente do Arenito da formação Aquidauana.

Ao longo da topossequência, foram abertas quatro trincheiras, localizadas em pontos distintos, a saber: terço superior de elevação (P1, altitude de $192 \mathrm{~m}$, $20^{\circ} 27^{\prime} 10$ " S e $55^{\circ} 40^{\prime} 01^{\prime}$ ” W), terço médio (P2, altitude de $166 \mathrm{~m}, 20^{\circ} 26$ ' 57 " S e $55^{\circ} 40$ ' 17 ” W), terço inferior (P3, altitude de $168 \mathrm{~m}, 20^{\circ} 27$ ' 13 " S e $55^{\circ} 40$ ' 25 ” W) e sopé (P4, altitude de $151 \mathrm{~m}$, $20^{\circ} 27^{\prime} 32$ " S e $55^{\circ} 40$ ' $38^{\prime}$ 'W) de uma encosta de aproximadamente $1.000 \mathrm{~m}$ de comprimento, com declives entre 3 e $8 \mathrm{~cm} \mathrm{~m}^{-1}$ e relevo suave ondulado. Quanto à drenagem, os perfis foram classificados como: bem drenado (P1), mal drenado (P2) e imperfeitamente drenado (P3 e P4). Os perfis foram descritos morfologicamente, segundo Santos et al. (2006), sendo coletadas amostras em todos os seus horizontes. As amostras após a coleta foram secas e peneiradas $(2 \mathrm{~mm})$, constituindo a terra fina seca ao ar (TFSA). Na TFSA, os atributos físicos e químicos foram determinados de acordo com Embrapa (1997). A composição granulométrica foi avaliada pelo método da pipeta, usando-se dispersão com $\mathrm{NaOH} 1 \mathrm{~mol} \mathrm{~L}^{-1}$; a densidade das partículas $(\mathrm{Dp})$ foi determinada pelo método do balão volumétrico (Embrapa, 1997).

$\mathrm{O} \mathrm{pH}$ foi determinado em água e $\mathrm{KCl} 1 \mathrm{~mol} \mathrm{~L}^{-1}$

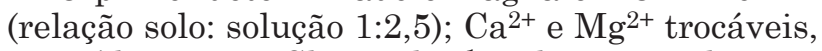
extraídos com $\mathrm{KCl} 1 \mathrm{~mol} \mathrm{~L}^{-1}$ e determinados por complexiometria;; $\mathrm{Na}^{+}$e $\mathrm{K}^{+}$trocáveis, extraídos por solução Mehlich-1 e determinados por fotometria de chama; $\mathrm{Al}^{3+}$ trocável, extraído com solução de $\mathrm{KCl}$ $1 \mathrm{~mol} \mathrm{~L}^{-1}$ e determinado por titulação; $\mathrm{H}+\mathrm{Al}$, extraídos com solução de acetato de Ca $0,5 \mathrm{~mol} \mathrm{~L}^{-1}$ tamponado a $\mathrm{pH}$ 7,0 e determinados por titulação; $\mathrm{P}$ disponível, extraído com solução de Mehlich-1 e determinado por colorimetria; e C orgânico total, oxidação com dicromato de potássio em meio sulfúrico.

Nos horizontes diagnósticos superficiais e subsuperficiais, os teores de $\mathrm{Si}, \mathrm{Al}, \mathrm{Fe}$ e Ti foram determinados após digestão ácida (método do ataque sulfúrico), a partir dos quais foram calculados os índices Ki e Kr (Camargo et al., 1986).

Simultaneamente à descrição morfológica dos perfis, amostras indeformadas de solo foram coletadas com auxílio de anel volumétrico com volume de $73,5 \mathrm{~cm}^{3}$, para determinação da densidade do solo (Ds), porosidade e resistência do solo à penetração. A Ds, a porosidade total, a macro e microporosidade foram determinadas utilizando-se um anel volumétrico em mesa de tensão, segundo Embrapa (1997). A resistência do solo à penetração foi quantificada em amostras saturadas, após $24 \mathrm{~h}$ em mesa de tensão e após $48 \mathrm{~h}$ em estufa a $105^{\circ} \mathrm{C}$, utilizando-se penetrógrafo de bancada modelo MA-933.

A partir dos atributos morfológicos e dos dados de análises físicas e químicas, os perfis de solo foram classificados segundo o Sistema Brasileiro de Classificação de Solos (SiBCS) (Embrapa, 2006).

\section{RESULTADOS E DISCUSSÃO}

\section{Aspectos morfológicos}

Em todos os perfis, nos horizontes mais superficiais predominou a estrutura do tipo granular (Quadro 1). Nos horizontes subsuperficiais foi verificada maior expressão de estrutura do tipo blocos subangulares no P1, blocos angulares no P2 e P3 e variações desses dois tipos de estrutura no $\mathrm{P} 4$.

Verificou-se o predomínio do matiz 5YR, sendo exceção o horizonte A2 do P1, que apresentou matiz 7,5YR, e de baixos valores do croma (valores entre $1 \mathrm{e}$ 
2), decorrentes dos maiores teores de matéria orgânica nesses horizontes. Nos horizontes subsuperficiais observou-se maior expressão de matizes mais avermelhados 2,5YR no P1 e P3 e 5,0 YR no P2 e P4. Em P3 verificaram-se cores com mosqueados vermelhos em quantidade abundante, tamanho médio e contraste distinto, enquanto em P2 e P4 foram verificados mosqueados vermelho-amarelados em quantidade abundante, tamanho pequeno e contraste distinto. Essas cores são indicativas da ocorrência do material plíntico (Embrapa, 2006).

A consistência, quando seca, variou de ligeiramente dura a dura no P1, dura a macia no P2, dura em todos os horizontes do P3 e de ligeiramente a extremamente dura em profundidade em P4. Quando úmida, a consistência analisada nos horizontes esteve entre muito friável e friável no $\mathrm{P} 1$; de friável a firme no P2 e P3; e friável a extremamente firme no P4. De maneira geral, em todos os perfis estudados, a consistência, quando molhada, foi ligeiramente plástica e ligeiramente pegajosa em superfície e plástica e ligeiramente pegajosa nos horizontes subsuperficiais.

\section{Atributos físicos}

Em todos os perfis os teores de argila foram inferiores a $280 \mathrm{~g} \mathrm{~kg}^{-1}$, em razão da natureza do material de origem (Quadro 2). No P1 e P3 observaram-se maiores teores de argila nos horizontes subsuperficiais (B), porém apenas no P1 foi identificado gradiente textural de 1,88, indicando, segundo os critérios estabelecidos pelo SiBCS (Embrapa, 2006), um horizonte B textural. No P3 o gradiente textural de 1,22, não foi suficiente para identificação deste horizonte. O grau de floculação nos perfis estudados foi baixo, sendo inferior a $50 \%$, exceto nos horizontes subsuperficiais $\left(\mathrm{Bt}_{2}, \mathrm{Bt}_{3}\right.$ e $\left.\mathrm{BC}\right)$ do $\mathrm{P} 1$.

Os valores de densidade do solo (Ds) variaram de 1,18 a $1,65 \mathrm{~kg} \mathrm{dm}^{-3}$ em P4 e P3, respectivamente, não tendo sido verificada correspondência entre os valores mais elevados de Ds e os de areia ou argila, ou de plintita ou petroplintita, observadas em P3 (Quadro 3). A porosidade total variou de 30 a $66 \%$ em P3 e P1, respectivamente, acompanhando as variações observadas na Ds. A microporosidade oscilou de 0,15

Quadro 1. Principais atributos morfológicos de solos desenvolvidos de arenito da formação Aquidauana- MS

\begin{tabular}{|c|c|c|c|c|c|c|c|c|}
\hline \multirow{2}{*}{ Horiz. } & \multirow{2}{*}{ Prof. } & \multicolumn{2}{|c|}{ Cor Munsell } & \multirow{2}{*}{ Estrutura $^{(2)}$} & \multicolumn{3}{|c|}{ Consistência } & \multirow{2}{*}{ Textura } \\
\hline & & Matriz & Mosqueado $^{(1)}$ & & Seca $^{(3)}$ & Úmida $^{(4)}$ & Molhada $^{(5)}$ & \\
\hline \multicolumn{8}{|c|}{ Perfil P1 - Terço superior da encosta } & \\
\hline $\mathrm{A} 1$ & $0-10$ & $7,5 \mathrm{YR} 3 / 2$ & & $\mathrm{~F}, \mathrm{P}, \mathrm{G}$ & $\mathrm{LD}$ & $\mathrm{MF} / \mathrm{F}$ & LPL e LPe & Franco-arenosa \\
\hline $\mathrm{A} 2$ & $10-24$ & 5 YR $3 / 2$ & & $\mathrm{M}, \mathrm{P}, \mathrm{G}$ & $\mathrm{D}$ & $\mathrm{MF} / \mathrm{F}$ & LPL e LPe & Franco-arenosa \\
\hline $\mathrm{AB}$ & $24-36$ & $5 Y R 3 / 3$ & & M, MP, BS & $\mathrm{D}$ & $\mathrm{F}$ & PL e LPe & Franco-arenosa \\
\hline BA & $36-50$ & $2,5 Y R 3 / 4$ & & $\mathrm{M}, \mathrm{P}, \mathrm{BS}$ & $\mathrm{LD}$ & $\mathrm{F}$ & PL e LPe & Franca-arenosa/franco-argiloarenosa \\
\hline Bt1 & $50-65$ & $2,5 \mathrm{YR} 4 / 4$ & & $\mathrm{M}, \mathrm{P}, \mathrm{BS}$ & LD & $\mathrm{F}$ & PL e LPe & Franco-argilo-arenosa \\
\hline Bt2 & $65-80$ & $2,5 \mathrm{YR} 4 / 4$ & & $\mathrm{M}, \mathrm{P}, \mathrm{BS}$ & $\mathrm{LD} / \mathrm{D}$ & $\mathrm{F}$ & PL e LPe & Franco-argilo-arenosa \\
\hline Bt3 & $80-109$ & $2,5 \mathrm{YR} 4 / 4$ & & $\mathrm{M}, \mathrm{P}, \mathrm{BS}$ & LD & $\mathrm{F}$ & PL e LPe & Franco-argilo-arenosa \\
\hline $\mathrm{BC}$ & $109-130^{+}$ & $2,5 \mathrm{YR} 4 / 4$ & & $\mathrm{M}, \mathrm{P}, \mathrm{BS}$ & LD & $\mathrm{F}$ & PL e LPe & Franco-argilo-arenosa \\
\hline \multicolumn{9}{|c|}{ Perfil P2 - Terço médio da encosta } \\
\hline Ap & $0-10$ & $5 Y R 3 / 1$ & & $\mathrm{M}, \mathrm{P}, \mathrm{G}$ & $\mathrm{D}$ & $\mathrm{F}$ & LPL e LPe & Franca \\
\hline BA & $10-17$ & $5 Y R 3 / 1$ & & $\mathrm{M}, \mathrm{MP}, \mathrm{BS} / \mathrm{G}$ & $\mathrm{D}$ & $\mathrm{Fr}$ & $\mathrm{PL}$ e $\mathrm{LPe}$ & Franco-arenosa \\
\hline $\mathrm{Bg} 1$ & $17-34$ & 5 YR $3 / 1$ & 5YR 3/2 Ab. Peq. Dis & $\mathrm{M}, \mathrm{MP}, \mathrm{BA}$ & $\mathrm{D}$ & $\mathrm{Fr}$ & PL e LPe & Franca \\
\hline $\mathrm{Bg} 2$ & $34-50$ & $5 Y R 3 / 1$ & 5YR $3 / 2$ Ab. Peq. Dis & $\mathrm{M}, \mathrm{MP}, \mathrm{BA}$ & $\mathrm{D}$ & Fr & PL e LPe & Franco-arenosa \\
\hline Bg3 & $50-70$ & $5 \mathrm{YR} 3 / 1$ & 5YR 3/2 Ab. Peq. Dis & $\mathrm{M}, \mathrm{P}, \mathrm{BA}$ & $\mathrm{D}$ & $\mathrm{Fr}$ & PL e LPe & Franco-arenosa \\
\hline $\mathrm{Bg} 4$ & $70-93$ & $5 \mathrm{YR} 4 / 2$ & 5YR $3 / 2$ Ab. Peq. Dis & $\mathrm{M}, \mathrm{P} / \mathrm{M}, \mathrm{BA}$ & $\mathrm{M}$ & $\mathrm{F}$ & PL e LPe & Areia-franca \\
\hline Bg5 & $93-110$ & 5 YR $4 / 2$ & 5YR $3 / 2$ Ab. Peq. Dis & $\mathrm{M}, \mathrm{P} / \mathrm{M}, \mathrm{BA}$ & $\mathrm{M}$ & $\mathrm{F}$ & PL e LPe & Franco-arenosa \\
\hline Bg6 & $110-127+$ & $5 \mathrm{YR} 4 / 2$ & 5YR $3 / 2$ Ab. Peq. Dis & $\mathrm{M}, \mathrm{P} / \mathrm{M}, \mathrm{BA}$ & M & $\mathrm{F}$ & PL e LPe & Franco-arenosa \\
\hline \multicolumn{9}{|c|}{ Perfil P3 - Terço inferior da encosta } \\
\hline A & $0-13$ & $5 Y R 3 / 2$ & & $\mathrm{M}, \mathrm{P}, \mathrm{G}$ & $\mathrm{D}$ & $\mathrm{F}$ & LPL e LPe & Franco-arenosa \\
\hline $\mathrm{AB}$ & $13-23$ & $5 Y R 3 / 3$ & & $\mathrm{M}, \mathrm{P}, \mathrm{BS}$ & $\mathrm{D}$ & $\mathrm{F}$ & PL e LPe & Franco-arenosa/franco-argiloarenosa \\
\hline Bf1 & $23-45$ & $2,5 \mathrm{YR} 3 / 4$ & 2,5YR 4/8 Ab. Med. Dis & $\mathrm{M}, \mathrm{P} / \mathrm{M}, \mathrm{BA}$ & $\mathrm{D}$ & $\mathrm{Fr}$ & $\mathrm{PL}$ e $\mathrm{Pe}$ & Franco-arenosa/franco-argiloarenosa \\
\hline Bf2 & $45-68$ & $2,5 \mathrm{YR} 3 / 6$ & 2,5YR 4/8 Ab. Med. Dis & $\mathrm{M}, \mathrm{P} / \mathrm{M}, \mathrm{BA}$ & $\mathrm{D}$ & $\mathrm{F}$ & PL e Pe & Franco-argilo-arenosa \\
\hline Bf3 & $68-90$ & $2,5 \mathrm{YR} 3 / 6$ & 2,5YR 4/8 Ab. Med. Dis & $\mathrm{M}, \mathrm{P}, \mathrm{BA}$ & $\mathrm{D}$ & $\mathrm{F}$ & LPL e LPe & Franco-arenosa/franco-argiloarenosa \\
\hline Bf4 & $90-140^{+}$ & $2,5 \mathrm{YR} 3 / 6$ & 2,5YR 4/8 Ab. Med. Dis & $\mathrm{M}, \mathrm{P}, \mathrm{BA}$ & $\mathrm{D}$ & $\mathrm{F}$ & PL e LPe & Franco-argilo-arenosa \\
\hline \multicolumn{9}{|c|}{ Perfil P4 - Sopé da encosta } \\
\hline $\mathrm{A} 1$ & $0-13$ & $5 Y R 3 / 1$ & & $\mathrm{M}, \mathrm{P} / \mathrm{M}, \mathrm{G}$ & $\mathrm{LD}$ & $\mathrm{F}$ & LPL e Pe & Franco-arenosa \\
\hline $\mathrm{A} 2$ & $13-29$ & 5 YR $3 / 2$ & & $\mathrm{M}, \mathrm{P}, \mathrm{BS}$ & $\mathrm{LD}$ & $\mathrm{F}$ & LPL e Pe & Franco-arenosa \\
\hline $\mathrm{BA}$ & $29-48$ & $7,5 \mathrm{YR} 3 / 4$ & & $\mathrm{M}, \mathrm{P}, \mathrm{BS}$ & $\mathrm{LD} / \mathrm{D}$ & $\mathrm{F}$ & LPL e LPe & Franco-arenosa \\
\hline $\mathrm{Bi1}$ & $48-67$ & $7,5 \mathrm{YR} 3 / 4$ & 5YR $3 / 2$ Ab. Peq. Dis & $\mathrm{M}, \mathrm{P}, \mathrm{BA} / \mathrm{BS}$ & $\mathrm{D}$ & $\mathrm{F}$ & $\mathrm{PL}$ e $\mathrm{Pe}$ & Franco-arenosa \\
\hline $\mathrm{Bi} 2$ & $67-80$ & $5 Y R 6 / 3$ & 5YR $3 / 2$ Ab. Peq. Dis & $\mathrm{M}, \mathrm{P}, \mathrm{BA}$ & MD & $\mathrm{F}$ & $\mathrm{PL}$ e $\mathrm{Pe}$ & Franco-arenosa \\
\hline $\mathrm{Bi} 3$ & $80-100^{+}$ & $5 Y R 6 / 3$ & 5YR $3 / 2 \mathrm{Ab}$. Peq. Dis & $\mathrm{M}, \mathrm{P}, \mathrm{BA}$ & $\mathrm{ED}$ & $\mathrm{EFr}$ & LPL e LPe & Franco-arenosa \\
\hline
\end{tabular}

(1) Ab: abundante; Peq: pequeno; Med: médio; Dis: distinto. ${ }^{(2)}$ Estrutura: grau de desenvolvimento (F: fraco M: moderado), tamanho (MP: muito pequeno, P: pequeno, M: médio), tipo (G: granular, BS: blocos subangulares, BA: blocos angulares). ${ }^{(3)}$ Consistência no estado seco (M: macia, LD: ligeiramente dura, D: dura, MD: muito dura, ED: extremamente dura). (4) Consistência no estado úmido (MF: muito friável, F: friável, Fr: firme, EFr: extremamente firme). ${ }^{(5)}$ Consistência no estado molhado (PL: plástico, LPL: ligeiramente plástica, LPe: ligeiramente pegajosa, Pe: pegajosa). 
Quadro 2. Granulometria, argila dispersa em água, grau de floculação e relação silte/argila de solos desenvolvidos de arenito da formação Aquidauana- MS

\begin{tabular}{|c|c|c|c|c|c|c|c|c|}
\hline \multirow{2}{*}{ Horizonte } & \multirow{2}{*}{ Profundidade } & \multicolumn{4}{|c|}{ Granulometria } & \multirow{2}{*}{$\begin{array}{c}\text { Argila dispersa } \\
\text { em água }\end{array}$} & \multirow{2}{*}{$\begin{array}{l}\text { Grau de } \\
\text { floculação }\end{array}$} & \multirow{2}{*}{ Silte/argila } \\
\hline & & Areia grossa & Areia fina & Silte & Argila & & & \\
\hline & \multirow[t]{2}{*}{$\mathrm{cm}$} & \multicolumn{5}{|c|}{$\mathrm{g} \mathrm{kg}^{-1} \mathrm{TFSA}$} & \multirow[t]{2}{*}{$\%$} & \\
\hline & & & Perfil P1 - Te & ço supe & ior da enc & & & \\
\hline $\mathrm{A} 1$ & $0-10$ & 340 & 450 & 110 & 100 & 90 & 10 & 1,10 \\
\hline $\mathrm{A} 2$ & $10-24$ & 330 & 440 & 110 & 120 & 100 & 17 & 0,92 \\
\hline $\mathrm{AB}$ & $24-36$ & 310 & 410 & 100 & 180 & 170 & 5 & 0,56 \\
\hline $\mathrm{BA}$ & $36-50$ & 240 & 410 & 150 & 200 & 190 & 5 & 0,75 \\
\hline Bt1 & $50-65$ & 240 & 370 & 140 & 250 & 200 & 20 & 0,56 \\
\hline Bt2 & $65-80$ & 210 & 370 & 140 & 280 & 50 & 83 & 0,14 \\
\hline Bt3 & $80-109$ & 230 & 370 & 130 & 270 & 30 & 89 & 0,48 \\
\hline \multirow[t]{2}{*}{$\mathrm{BC}$} & $109-130+$ & 220 & 410 & 140 & 240 & 30 & 87 & 0,58 \\
\hline & \multicolumn{7}{|c|}{ Perfil P2 - Terço médio da encosta } & \\
\hline Ap & $0-10$ & 170 & 290 & 360 & 180 & 160 & 12 & 2,0 \\
\hline $\mathrm{BA}$ & $10-17$ & 210 & 320 & 300 & 170 & 140 & 18 & 1,76 \\
\hline $\mathrm{Bg} 1$ & $17-34$ & 180 & 260 & 370 & 190 & 130 & 32 & 1,95 \\
\hline Bg2 & $34-50$ & 270 & 350 & 240 & 140 & 130 & 7 & 1,71 \\
\hline Bg3 & $50-70$ & 250 & 370 & 220 & 150 & 140 & 7 & 1,46 \\
\hline $\mathrm{Bg} 4$ & $70-93$ & 320 & 330 & 220 & 130 & 90 & 31 & 0,08 \\
\hline Bg5 & $93-110$ & 230 & 310 & 350 & 100 & 90 & 10 & 3,50 \\
\hline \multirow[t]{2}{*}{ Bg6 } & $110-127+$ & 190 & 360 & 340 & 110 & 90 & 90 & 3,09 \\
\hline & \multicolumn{7}{|c|}{ Perfil P3 - Terço inferior da encosta } & \\
\hline A & $0-13$ & 380 & 380 & 120 & 120 & 90 & 25 & 1,00 \\
\hline $\mathrm{AB}$ & $13-23$ & 260 & 380 & 160 & 200 & 160 & 20 & 0,80 \\
\hline $\mathrm{Bf}$ & $23-45$ & 285 & 360 & 165 & 190 & 155 & 20 & 0,86 \\
\hline Bf2 & $45-68$ & 240 & 380 & 175 & 200 & 125 & 36 & 0,87 \\
\hline Bf3 & $68-90$ & 215 & 415 & 175 & 200 & 135 & 32 & 0,87 \\
\hline \multirow[t]{2}{*}{ Bf4 } & $90-140+$ & 237 & 370 & 187 & 190 & 160 & 17 & 0,98 \\
\hline & \multicolumn{7}{|c|}{ Perfil P4 - Sopé da encosta } & \\
\hline $\mathrm{A} 1$ & $0-13$ & 60 & 490 & 340 & 110 & 70 & 36 & 3,09 \\
\hline A2 & $13-29$ & 80 & 510 & 310 & 100 & 80 & 20 & 3,10 \\
\hline $\mathrm{BA}$ & $29-48$ & 120 & 460 & 290 & 120 & 110 & 8 & 2,41 \\
\hline Bi1 & $48-67$ & 180 & 390 & 300 & 130 & 120 & 8 & 2,31 \\
\hline Bi2 & $67-80$ & 300 & 300 & 280 & 110 & 80 & 27 & 2,55 \\
\hline $\mathrm{Bi} 3$ & $80-100+$ & 310 & 300 & 300 & 80 & 80 & 0 & 3,75 \\
\hline
\end{tabular}

a $0,26 \mathrm{~cm}^{3} \mathrm{~cm}^{-3}$ no $\mathrm{P} 4$ e P3, respectivamente, enquanto a resistência à penetração (RP) acompanhou as variações observadas para a consistência do solo. Nas amostras saturadas, os valores variaram de $0 \mathrm{MPa}$ (P2 e P4) a 0,77 MPa (P3), com tendência a valores próximos de zero nas maiores profundidades dos perfis de drenagem imperfeita (P2 e P4). Simulando a umidade próxima à capacidade de campo, observaramse valores de RP variando de 0,14 a 1,54 MPa em P1 e P2, respectivamente, estando a consistência, quando úmida, entre friável e firme nos referidos perfis (Quadro 1). A consistência, quando seca, apresentou variação de $\mathrm{RP}$ de 1,28 a 15,15 MPa, em P4 e P1, respectivamente. A resistência à penetração associada a algumas características morfológicas do solo, como a consistência, pode estar diretamente relacionada ao crescimento radicular das plantas. Desse modo, Lipiec \& Hatano (2003) afirmam que valores de RP variando de 1,0 a 1,7 MPa começam a restringir o crescimento radicular. Nos perfis estudados, as amostras, quando na umidade correspondente à capacidade de campo, não ultrapassaram o limite crítico de 2,0 MPa, preconizado por Beutler et al. (2003).

\section{Atributos químicos}

Os valores de $\mathrm{pH}$ em água variaram entre $4,2 \mathrm{e}$ 6,5 (Quadro 4). No P1, observaram-se os menores valores, os quais reduzem em profundidade, paralelamente ao aumento dos teores de $\mathrm{Al}^{3+}$. Nos demais perfis (P2, P3 e P4), os valores de pH em água foram próximos a 6,0 , com pouca variação em profundidade, não sendo observada a presença de $\mathrm{Al}^{3+}$.

Os maiores teores de $\mathrm{Ca}^{2+}$ ocorreram nos horizontes superficiais, com variações de 0,1 a $0,8 \mathrm{cmol}_{\mathrm{c}} \mathrm{kg}^{-1}, 2,4$ a $5,8 \mathrm{cmol}_{\mathrm{c}} \mathrm{kg}^{-1}, 0,8$ a $1,4 \mathrm{cmol}_{\mathrm{c}} \mathrm{kg}^{-1}$ e 0,0 a $2,6 \mathrm{cmol}_{\mathrm{c}} \mathrm{kg}^{-1}$, no P1, P2, P3 e P4, respectivamente (Quadro 4). Os valores de $\mathrm{Mg}^{2+}$ no P1 (0,1 a $\left.0,6 \mathrm{cmol}_{\mathrm{c}} \mathrm{kg}^{-1}\right)$ e P4 (0,4 a 1,1 $\left.\mathrm{cmol}_{\mathrm{c}} \mathrm{kg}^{-1}\right)$ apresentaram pouca variação em profundidade. Já no P2 os valores 
Quadro 3. Densidade, porosidade e resistência à penetração de solos desenvolvidos de arenito da formação Aquidauana-MS

\begin{tabular}{|c|c|c|c|c|c|c|c|c|c|}
\hline \multirow{2}{*}{ Horizonte } & \multirow{2}{*}{ Profundidade } & \multicolumn{2}{|c|}{ Densidade } & \multicolumn{3}{|c|}{ Porosidade } & \multicolumn{3}{|c|}{ Resistência à penetração } \\
\hline & & Solo & Partícula & Macro & Micro & Total & SAT & $\mathbf{C C}$ & $105^{\circ} \mathrm{C}$ \\
\hline & $\mathrm{cm}$ & \multicolumn{2}{|c|}{$-\mathrm{kg} \mathrm{dm}^{-3}-$} & \multicolumn{3}{|c|}{$\%$} & \multicolumn{3}{|c|}{$\longrightarrow \mathrm{MPa}$} \\
\hline \multicolumn{10}{|c|}{ Perfil P1 - Terço superior da encosta } \\
\hline A1 & $0-10$ & 1,63 & 2,84 & 20,9 & 18,7 & 39,7 & 0,36 & 0,54 & 7,40 \\
\hline $\mathrm{A} 2$ & 1024 & 1,46 & 2,81 & 23,9 & 16,6 & 40,6 & 0,01 & 0,36 & 11,41 \\
\hline $\mathrm{AB}$ & $24-36$ & 1,57 & 2,84 & 20,1 & 18,2 & 38,3 & 0,20 & 0,41 & 13,92 \\
\hline BA & $36-50$ & 1,49 & 2,84 & 22,3 & 19,2 & 41,6 & 0,09 & 0,26 & 6,52 \\
\hline Bt1 & $50-65$ & 1,46 & 2,79 & 23,3 & 22,0 & 45,3 & 0,12 & 0,33 & 7,80 \\
\hline Bt2 & $65-80$ & 1,46 & 2,79 & 20,2 & 21,5 & 41,7 & 0,30 & 0,56 & 10,74 \\
\hline Bt3 & $80-109$ & 1,52 & 2,75 & 17,2 & 22,3 & 39,5 & 0,44 & 0,61 & 15,15 \\
\hline $\mathrm{BC}$ & $109-130+$ & 1,25 & 2,84 & 45,7 & 20,3 & 65,9 & 0,01 & 0,14 & 2,11 \\
\hline \multicolumn{10}{|c|}{ Perfil P2 - Terço médio da encosta } \\
\hline Ap & $0-10$ & 1,36 & 2,85 & 24,8 & 18,6 & 43,4 & 0,02 & 0,82 & 3,13 \\
\hline BA & 1017 & 1,58 & 2,85 & 20,8 & 19,9 & 40,6 & 0,72 & 1,54 & 10,94 \\
\hline $\mathrm{Bg} 1$ & $17-34$ & 1,40 & 2,65 & 20,3 & 25,2 & 45,5 & 0,48 & 0,98 & 7,13 \\
\hline Bg2 & $34-50$ & 1,53 & 2,49 & 15,1 & 21,3 & 36,4 & 0,30 & 1,54 & 12,65 \\
\hline Bg3 & $50-70$ & 1,63 & 2,83 & 25,6 & 17,3 & 42,9 & 0,07 & 0,65 & 6,54 \\
\hline $\mathrm{Bg} 4$ & $70-93$ & 1,47 & 2,87 & 19,2 & 18,8 & 38,0 & 0,00 & 0,41 & 2,49 \\
\hline Bg5 & $93-110$ & 1,57 & 2,61 & 16,6 & 18,9 & 35,5 & 0,00 & 0,59 & 11,78 \\
\hline Bg6 & $110-127+$ & 1,40 & 2,57 & 18,2 & 18,8 & 37,1 & 0,00 & 0,59 & 5,94 \\
\hline \multicolumn{10}{|c|}{ Perfil P3 - Terço inferior da encosta } \\
\hline A & $0-13$ & 1,50 & 2,80 & 16,3 & 21,4 & 37,8 & 0,24 & 0,56 & 10,34 \\
\hline $\mathrm{AB}$ & $13-23$ & 1,36 & 2,88 & 13,9 & 16,1 & 30,1 & 0,38 & 0,58 & 7,86 \\
\hline $\mathrm{Bf}$ & $23-45$ & 1,65 & 2,83 & 15,8 & 25,8 & 41,5 & 0,50 & 0,43 & 10,61 \\
\hline $\mathrm{Bf} 2$ & $45-68$ & 1,57 & 2,81 & 17,5 & 22,4 & 40,0 & 0,32 & 0,86 & 9,18 \\
\hline Bf3 & $68-90$ & 1,46 & 2,87 & 20,7 & 20,5 & 41,1 & 0,16 & 0,42 & 6,80 \\
\hline Bf4 & $90-140+$ & 1,58 & 2,82 & 18,97 & 21,1 & 40,1 & 0,31 & 0,53 & 8,76 \\
\hline \multicolumn{10}{|c|}{ Perfil P4 - Sopé da encosta } \\
\hline A1 & $0-13$ & 1,18 & 2,67 & 35,0 & 15,0 & 50,0 & 0,04 & 0,49 & 1,28 \\
\hline $\mathrm{A} 2$ & $13-29$ & 1,39 & 2,72 & 20,4 & 19,9 & 40,2 & 0,13 & 0,67 & 10,36 \\
\hline $\mathrm{BA}$ & $29-48$ & 1,36 & 2,72 & 30,2 & 17,5 & 47,7 & 0,00 & 0,41 & 4,19 \\
\hline Bi1 & $48-67$ & 1,37 & 2,80 & 26,0 & 17,9 & 43,8 & 0,00 & 0,34 & 4,12 \\
\hline $\mathrm{Bi} 2$ & $67-80$ & 1,49 & 2,84 & 25,1 & 17,5 & 42,6 & 0,00 & 0,96 & 9,12 \\
\hline $\mathrm{Bi} 3$ & $80-100+$ & 1,41 & 2,93 & 20,9 & 16,0 & 36,9 & 0,11 & 0,54 & 11,57 \\
\hline
\end{tabular}

Dp: densidade da partícula; Ds: densidade do solo; SAT: amostra saturada; CC: umidade simulada na capacidade de campo; $105{ }^{\circ} \mathrm{C}$ : amostra em estufa a $105^{\circ} \mathrm{C}$.

de $\mathrm{Mg}^{2+}$ diminuíram em profundidade, variando de 0,6 a $2,1 \mathrm{cmol}_{\mathrm{c}} \mathrm{kg}^{-1}$, enquanto no P3 o padrão foi inverso, ou seja, os valores de $\mathrm{Mg}^{2+}$ aumentaram em profundidade. Ainda, nos horizontes subsuperficiais dos perfis $\mathrm{P} 3$ e $\mathrm{P} 4$, os valores de $\mathrm{Mg}^{2+}$ foram maiores que os de $\mathrm{Ca}^{+2}$. Estudando Plintossolos de origem da formação Itapecuru, no Estado do Maranhão, Anjos et al. (2007) também verificaram esse padrão, o qual atribuíram à contribuição de maiores teores de $\mathrm{Mg}$ dos sedimentos, bem como à maior solubilidade do $\mathrm{Mg}$ em relação ao Ca.

Os valores de CTC variaram de $1,6 \mathrm{cmol}_{\mathrm{c}} \mathrm{kg}^{-1}$ no P4 a 10,3 $\mathrm{cmol}_{\mathrm{c}} \mathrm{kg}^{-1}$ no P2 (Quadro 4), com predomínio dos íons de hidrogênio no P1 e P4 e de Ca no P2 e P3. Quanto ao valor V \%, no P1 e P4, os horizontes diagnósticos subsuperficiais foram identificados como distróficos $(\mathrm{V}<50 \%)$. Os perfis $\mathrm{P} 2$ e $\mathrm{P} 3$ apresentaram elevada saturação por bases (V > 50 \%) nos horizontes diagnósticos subsuperficiais, identificando o caráter eutrófico (Embrapa, 2006). À exceção do perfil P2 de horizonte $\mathrm{Bg} 2$, os demais apresentaram atividade da fração argila inferior a $27 \mathrm{cmol}_{\mathrm{c}} \mathrm{kg}^{-1}$, sendo caracterizados como de argila de baixa atividade (Quadro 5).

No P2, o teor de carbono orgânico (CO) foi mais elevado (18,9 $\left.\mathrm{g} \mathrm{kg}^{-1}\right)$ quando comparado ao dos demais perfis, o que pode estar relacionado à textura com maior teor de argila e silte, com valores de 180 e $360 \mathrm{~g} \mathrm{~kg}^{-1}$ de TFSA, respectivamente (Quadro 2). Nos demais perfis, os teores de CO foram baixos: 5,1, 4,8 e $7,0 \mathrm{~g} \mathrm{~kg}^{-1}$ no P1, P3 e P4, respectivamente, nos horizontes superficiais. Os maiores valores de $\mathrm{P}$ disponível foram observados nos horizontes superficiais do P2 (55 $\left.\mathrm{mg} \mathrm{kg}^{-1}\right)$, seguido do horizonte $\mathrm{A} 1$ do $\mathrm{P} 1\left(30 \mathrm{mg} \mathrm{kg}^{-1}\right)$; valores próximos à unidade foram observados no P3 e P4. Os teores elevados de P nos horizontes superficiais do P1 e P2 devem estar relacionados aos resíduos de adubações fosfatadas prática comum adotada para cultivo dessas áreas. 
Quadro 4. Atributos químicos, teor de carbono orgânico e fósforo de solos desenvolvidos de arenito da formação Aquidauana- MS

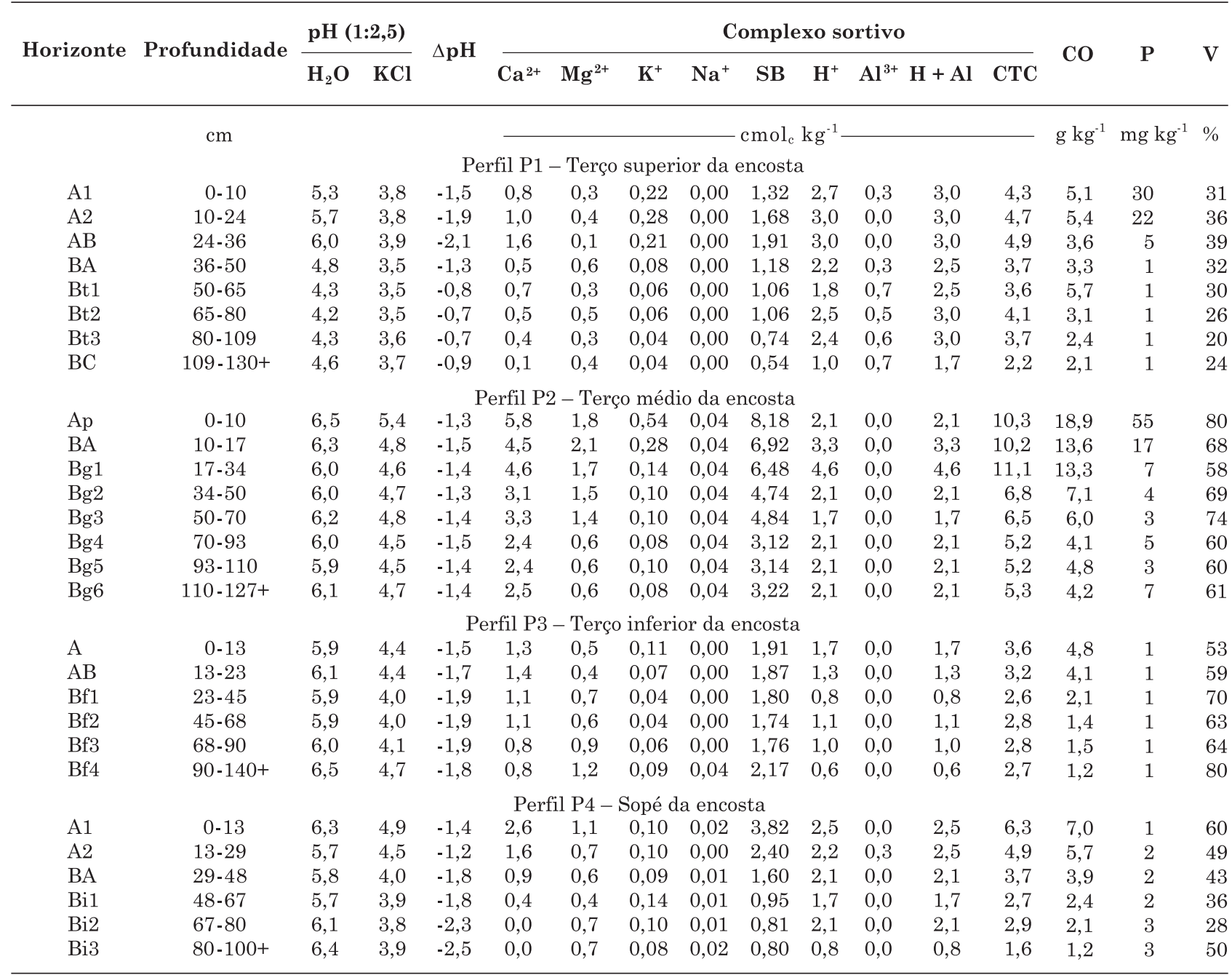

CO: carbono orgânico total; SB: soma de bases $\left(\mathrm{SB}=\mathrm{Ca}^{2+}+\mathrm{Mg}^{2+}+\mathrm{K}^{+}+\mathrm{Na}^{+}\right)$; $\mathrm{CTC}=(\mathrm{SB}+\mathrm{H}+\mathrm{Al}) ; \mathrm{V}=100 \mathrm{SB} / \mathrm{CTC}$.

Os valores de $\mathrm{SiO}_{2}$ variaram de 40 a $99 \mathrm{~g} \mathrm{~kg}^{-1}$; $\mathrm{Al}_{2} \mathrm{O}_{3}$, de 46 a $95 \mathrm{~g} \mathrm{~kg}^{-1} ; \mathrm{Fe}_{2} \mathrm{O}_{3}$, de 15 a $33 \mathrm{~g} \mathrm{~kg}^{-1}$; e $\mathrm{TiO}_{2}$, de 2 a $8 \mathrm{~g} \mathrm{~kg}^{-1}$ (Quadro 5). De maneira geral, os baixos valores desses elementos estão associados aos baixos conteúdos de argila observados nos solos estudados. No P1 e P3 verificaram-se incrementos nos teores de $\mathrm{SiO}_{2}, \mathrm{Al}_{2} \mathrm{O}_{3}, \mathrm{Fe}_{2} \mathrm{O}_{3}$ nos horizontes subsuperficiais. Para os perfis $\mathrm{P} 2$ e P4 esse padrão não foi verificado, devido, provavelmente, à localização na parte mais baixa da paisagem (P4) e ao relevo côncavo (P2), ocasionando drenagem imperfeita. Ainda no P2, observaram-se os maiores valores de Ki $(2,0$ e 2,1$)$ e $\mathrm{Kr}(1,7$ e 1,8$)$ - indicativo de solos com baixo grau de intemperismo (Embrapa, 2006). Em trabalho com Plintossolos no Cerrado, Gomes et al. (2007) observaram valores de $\mathrm{Ki}(0,7$ a 1,1$)$ e $\mathrm{Kr}(0,5$ a 0,9 ) inferiores aos encontrados no perfil P3 com a presença de plintita. Por outro lado, os mesmos autores observaram para Cambissolos valores de Ki
$(1,6$ a 1,8$)$ e $\operatorname{Kr}(0,9$ a 1,4$)$, corroborando os observados para o perfil P4. Para Plintossolos no Maranhão, Anjos et al. (2007) verificaram valores de Ki elevados, superiores a 2,2, indicando grau de intemperização não muito elevado dos solos por eles estudados.

Quanto aos teores de $\mathrm{TiO}_{2}$, observou-se pouca variação, podendo-se inferir, pela uniformidade dos materiais e pelo material de origem do solo, que se trata de arenito subjacente.

\section{Gênese e classificação dos solos}

Em função dos teores de $\mathrm{CO}$ e da espessura, os horizontes superficiais de todos os perfis foram identificados como A moderado (Embrapa, 2006). No terço superior da encosta, o P1 apresenta um horizonte de acumulação de argila em subsuperfície, identificado pela presença de gradiente textural de 1,88; a formação deste gradiente pode ser devido à ação do processo 
Quadro 5. Composição química da fração terra fina seca ao ar, Ki, Kr e atividade da fração argila em alguns horizontes de solos desenvolvidos de arenito da Formação Aquidauana-MS

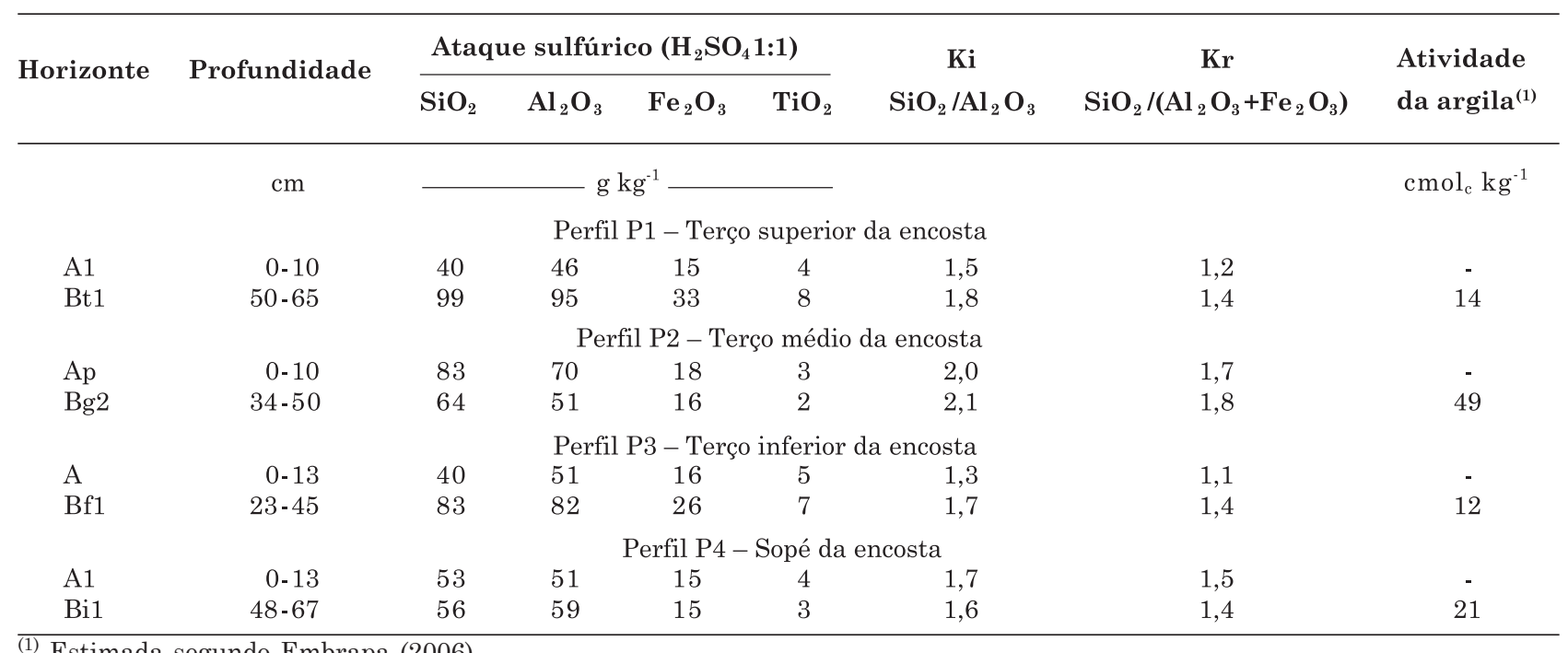

(1) Estimada segundo Embrapa (2006).

pedogenético de eluviação e iluviação associado à erosão seletiva de argila dos horizontes superficiais. A presença do horizonte $\mathrm{B}$ textural, associada à atividade de argila baixa (CTC $<27 \mathrm{cmol}_{\mathrm{c}} \mathrm{kg}^{-1}$ argila), faz com que esse perfil se enquadre na ordem dos Argissolos. Nos horizontes subsuperficiais, o predomínio do matiz 2,5 YR faz com que ele seja classificado, em nível de subordem, como Argissolo Vermelho. Como os horizontes subsuperficiais têm baixa saturação por bases ( $\mathrm{V}<50 \%$ ), enquadra-se como Argissolo Vermelho distrófico no nível categórico de Grande Grupo. Por não apresentar nenhum outro atributo diferencial, o perfil foi classificado, em nível de subgrupo, como Argissolo Vermelho distrófico típico.

No terço médio de paisagem, o solo situa-se numa zona ligeiramente deprimida, com maior influência do lençol freático, o que permite a acumulação de água por mais tempo, favorecendo o desenvolvimento do processo pedogenético de gleização, indicado pelas cores com baixo croma, associada à presença de mosqueados, que variam de 20 a $40 \%$. O conjunto de atributos observados faz com que o perfil seja classificado como Gleissolo Háplico, no nível de subordem. A atividade de argila baixa (CTC $<27 \mathrm{cmol}_{\mathrm{C}} \mathrm{kg}^{-1}$ argila), associada aos elevados valores de saturação por bases ( $\mathrm{V}>50 \%$ ), leva o perfil a ser enquadrado, em nível de subgrupo, como Gleissolo Háplico Tb eutrófico típico. Os elevados valores de saturação por bases podem ser decorrentes do transporte e acumulação das bases em função da depressão observada nesse ponto da paisagem.

O P3, no terço inferior de encosta, é submetido a uma maior influência das variações sazonais do lençol freático. Observa-se, nos seus horizontes subsuperficiais, mosqueamento abundante de coloração avermelhada, tamanho pequeno e contraste distinto. Associada à coloração mosqueada tem-se a presença de plintita e concreções ferruginosas, em quantidade variando de comum a abundante, correspondendo a valores entre 15 e $40 \%$ do volume do solo. Esses atributos indicam a ocorrência do processo pedogenético de plintitização, sendo diagnóstico do horizonte plíntico, segundo os critérios adotados pelo SiBCS (Embrapa, 2006). A partir dos atributos e horizontes identificados, o perfil foi classificado, em nível de subordem, como Plintossolo Háplico. Os elevados valores de saturação por bases (V > 50 \%) fazem com que o perfil seja classificado, em nível de subgrupo, como Plintossolo Háplico eutrófico típico. Os elevados valores de saturação por bases também podem ser resultantes do transporte e de posterior acumulação de íons nesse ponto de paisagem.

Para o P4, localizado no sopé da encosta, observaram-se mosqueados de coloração vermelhoamarelada, em quantidade abundante e contraste distinto. As cores observadas nos horizontes subsuperficiais não atendem aos requisitos definidos para horizonte glei. Nesse perfil não foi verificada a expressão de nenhum processo pedogenético em grande intensidade, sendo o horizonte diagnóstico subsuperficial identificado como B incipiente. Observou-se distribuição irregular dos teores de argila e da relação silte/argila em profundidade, o que faz com que ele seja classificado, em nível de subordem, como Cambissolo Flúvico. Os baixos valores de V \% e argila de atividade baixa (CTC $<27 \mathrm{cmol}_{\mathrm{c}} \mathrm{kg}^{-1}$ argila) fazem com que, na categoria de subgrupo, ele se enquadre como Cambissolo Flúvico Tb distrófico típico. 


\section{CONCLUSÕES}

1. Como principais atributos morfológicos e físicos, verificaram-se cores avermelhadas nos perfis localizados nas posições de paisagem condicionadoras de melhor drenagem e mosqueamento, associado a cores mais amareladas, naqueles localizados onde ocorre maior restrição à drenagem. Em todos os perfis, observou-se o predomínio da estrutura em blocos subangulares e textura variando de franco-arenosa a franco-argiloarenosa.

2. Quanto aos atributos químicos e mineralógicos, verificaram-se solos com saturação por bases variável e predomínio de argilas de atividade baixa (CTC $<27 \mathrm{cmol}_{\mathrm{c}} \mathrm{kg}^{-1}$ argila).

3. Por meio da análise dos atributos morfológicos, químicos, físicos e mineralógicos, constatou-se a influência dos fatores relevo e material de origem, como principais atuantes na gênese dos solos estudados.

\section{AGRADECIMENTOS}

Os autores agradecem à Fundação de Apoio ao Desenvolvimento do Ensino, Ciência e Tecnologia do Estado de Mato Grosso do Sul - FUNDECT, chamada Universal 02/2005, processo 41/100.114/2006; e ao CNPq projeto “casadinho", processo 620029/20080, pelo apoio financeiro.

\section{LITERATURA CITADA}

ANJOS, L.H.C.; PEREIRA, M.G.; PÉREZ, D.V. \& RAMOS, D.P. Caracterização e classificação de Plintossolos no município de Pinheiro-MA. R. Bras. Ci. Solo, 31:10351044, 2007.

BEUTLER, A.N.; SILVA, M.L.N.; CURI, N.; FERREIRA, M.M.; CRUZ, J.C. \& PEREIRA FILHO, I.A. Resistência à penetração e permeabilidade de Latossolo Vermelho distrófico típico sob sistemas de manejo na Região dos Cerrados. R. Bras. Ci. Solo, 25:167-177, 2003.
CAMARGO, O.A.; MONIZ, A.C.; JORGE, J.A. \& VALADARES, J.M.A.S. Métodos de análises química, mineralógica e física de solos do Instituto Agronômico de Campinas. Campinas, Instituto Agronômico de Campinas, 1986. 94p. (Boletim Técnico, 106)

COELHO, M.R. \& VIDAL-TORRADO, P. Caracterização e gênese de perfis plínticos desenvolvidos de arenito do grupo bauru. II- Mineralogia. R. Bras. Ci. Solo, 27:495507,2003

CURI, N. \& FRANZMEIER, D.P. Topossequence of Oxisols from the Central Plateau of Brazil. Soil Sci. Soc. Am., 48:341-346, 1984

EMPRESA BRASILEIRA DE PESQUISA AGROPECUÁRIA EMBRAPA. Centro Nacional de Pesquisa de Solos. Manual de métodos de análises de solo. 2.ed. Rio de Janeiro, 1997. 212p.

EMPRESA BRASILEIRA DE PESQUISA AGROPECUÁRIA EMBRAPA. Centro Nacional de Pesquisa de Solos. Sistema brasileiro de classificação de solos. Rio de Janeiro, 2006. $306 \mathrm{p}$.

GHIDIN, A.A.; MELO, V.F.; LIMA, V.C. \& LIMA, J.M.J.C. Topossequências de Latossolos originados de rochas basálticas no Paraná. II - Relação entre mineralogia da fração argila e propriedades físicas dos solos. R. Bras. Ci. Solo, 30:307-319, 2006.

GOMES, J.B.V.; CUR, N.; SCHULZE, D.G.; MARQUES, J.J.G.S.M.; KER, J.C. \& MOTTA, P.E.F. Mineralogia e micromorfologia de solos esqueléticos do bioma cerrado, no leste de Goiás. R. Bras. Ci. Solo, 31:875-886, 2007.

LIPIEC, J. \& HATANO, R. Quantification of compaction effects on soil physical properties and crop growth. Geoderma, 116:107-136, 2003.

MOTTA, P.E.F.; CARVALHO FILHO, A.; KER, J.C.; PEREIRA, N.R.; CARVALHO JÚNIOR, W. \& BLANCANEAUX, P. Relações solo-superfície geomórfica e evolução da paisagem em uma área do Planalto Central Brasileiro. Pesq. Agropec. Bras., 37:869-878, 2002.

RADAMBRASIL. FOLHA SF.21 CAMPO GRANDE. Ministério das Minas e Energia - Departamento Nacional da Produção Mineral. Levantamentos de Recursos Naturais. Rio de Janeiro, 1982. v.28.

SANTOS, R.D.; LEMOS, R.C.; SANTOS, H.G.; KER, J.C. \& ANJOS, L.H.C. Manual de descrição e coleta de solo no campo. 5.ed. Viçosa, MG, Sociedade Brasileira de Ciência do Solo, 2006. 92p. 